Originalien

Med Klin Intensivmed Notfmed 2022 · 117:289-296 https://doi.org/10.1007/s00063-021-00816-1

Eingegangen: 9. Januar 2021

Überarbeitet: 19. Februar 2021

Angenommen: 3. März 2021

Online publiziert: 20. April 2021

(c) Der/die Autor(en) 2021

Redaktion

M. Buerke, Siegen

\section{Hintergrund und Fragestellung}

Ausbrüche in Altenheimen und stationären Pflegeeinrichtungen stellen ein häufiges Phänomen während der Corona Virus Disease-2019(COVID-19)Pandemie dar und sind mit hoher Morbidität und Mortalität assoziiert [20, 24]. Während bis dato ein Massenanfall von Erkrankten (MANE) bzw. Infizierten (MANI) ein seltenes Ereignis im Rettungsdienst darstellte [25], sehen sich die Sanitätseinsatzleitungen (San-EL) aktuell wiederholt mit MANI-Lagen in stationären Pflegeeinrichtungen konfrontiert. Durch die gleichzeitige Erkrankung bzw. Quarantänepflicht von sowohl Bewohnern als auch Personal reichen die Konsequenzen solcher Ereignisse bis hin zum völligen Zusammenbruch der Betriebsbereitschaft der jeweiligen Einrichtung. Gleichzeitig finden diese Ausbrüche im Kontext einer reduzierten Aufnahmebereitschaft der Krankenhäuser statt. Die einsatztaktischen Vorgehensweisen für den Massenanfall von Verletzten (MANV) lassen sich nur eingeschränkt auf diese Einsatzlagen übertragen.

W. Schreiber ist Leitender Notarzt im Rettungsdienstbereich Amberg. M. Dittmar ist Ärztlicher Bezirksbeauftragter Rettungsdienst für den Rettungsdienstbezirk Oberpfalz.

Wolfgang Schreiber ${ }^{1}$ Philipp Wolf ${ }^{2}$ Nicole Bigalke ${ }^{1} \cdot$ Marc U. Bigalke $^{3}$. Bernhard M. Graf ${ }^{2} \cdot$ Michael S. Dittmar $^{2,4}$

${ }^{1}$ Zweckverband für Rettungsdienst und Feuerwehralarmierung Amberg, Amberg, Deutschland

${ }^{2}$ Klinik für Anästhesiologie, Universitätsklinikum Regensburg, Regensburg, Deutschland

${ }^{3}$ Zentrale Notaufnahme, Klinikum St. Marien Amberg, Amberg, Deutschland

${ }^{4}$ Sachgebiet 10, Regierung der Oberpfalz, Regensburg, Deutschland

\title{
Managementstrategie für den Massenanfall von Erkrankten/ Infizierten in Alten- und Pflegeheimen im Kontext der COVID-19-Pandemie
}

\section{Studiendesign und Unter- suchungsmethoden}

Auf Basis der Erfahrungen aus 2 MANIEinsätzen in Alten- bzw. Pflegeheimen stellen die Autoren ein Phasenmodell für das akute Management derartiger Ereignisse dar. Ergänzend wurde eine PubMed-Literaturrecherche mit den Medical Subject Headings "mass casualty incidents“, „emergency medical services“, "residential facilities“ und „disease outbreaks" sowie eine selektive Literatursuche durchgeführt.

\section{Ergebnisse}

\section{Fallbericht}

In einer stationären Pflegeeinrichtung mit 47 Bewohnern wird nach vereinzelten COVID-19-Fällen eine Reihentestung durchgeführt. Während der Testphase liefert der Rettungsdienst bereits 5 symptomatische Patienten in Kliniken ein.

\section{Tag 1 (Donnerstag)}

In der Testung erweisen sich 37 der 42 verbliebenen Heimbewohner sowie 17 Mitarbeiter als „Severe Acute Respiratory Syndrome Corona Virus-2“(SARSCoV-2)-positiv. Für weitere Beschäftigte wird als Kontaktpersonen der Kategorie I Quarantäne angeordnet. Dies führt da$\mathrm{zu}$, dass nur eine einzige Pflegekraft zur Spätschicht erscheint. Über diese Situation wird die Katastrophenschutzbehörde (Führungsgruppe Katastrophenschutz [FüGK]) informiert, die um $14.30 \mathrm{Uhr}$ die Entsendung der San-EL zur Lageerkundung veranlasst.

Der San-EL wird übergeben, dass mehrere Bewohner einen reduzierten Allgemeinzustand und Atembeschwerden aufwiesen. In einer ersten Lagemeldung werden daraufhin 2 Rettungstransportwägen (RTW), ein Notarzteinsatzfahrzeug (NEF) sowie die Unterstützungsgruppe San-EL angefordert.

In vollständiger Infektionsschutzausrüstung beginnen 3 Teams gegen 15.00 Uhr mit der Sichtung. Abweichend vom lokal etablierten Amberg-Schwandorf-Algorithmus für die Vorsichtung (ASAV; [12]) wird nach der COVID-19Entscheidungshilfe der ÄLRD Bayern gesichtet [23, 27]. Das Sichtungsergebnis wird zusammen mit Vitalparametern und Temperatur auf Patientenanhängekarten dokumentiert.

Die Sichtung ist nach $90 \mathrm{~min}$ abgeschlossen. Nachalarmierte Rettungsmittel übernehmen den Transport von 8 hospitalisierungspflichtigen Patienten (einmal Sichtungskategorie [SK] I, 7-mal 


\begin{tabular}{|c|c|c|}
\hline & MANV & MANI \\
\hline $\begin{array}{l}\text { Vorbe- } \\
\text { reitung }\end{array}$ & $\begin{array}{l}\text { Einsatzkonzepte etabliert, Übungs- und Einsatzerfahrung vor- } \\
\text { handen }\end{array}$ & Keine etablierten Konzepte, kaum Übungs- und Einsatzerfahrung \\
\hline $\begin{array}{l}\text { Infra- } \\
\text { struktur }\end{array}$ & $\begin{array}{l}\text { Krankenhäuser i.d. R. voll leistungsfähig. Betreuungsbedarf für } \\
\text { unverletzte Betroffene meist nur kurz }\end{array}$ & $\begin{array}{l}\text { Krankenhäuser bereits mit COVID-19-Patienten belastet. In Heimen } \\
\text { u.U. (aufwändigere) pflegerische Betreuung der verbleibenden Be- } \\
\text { wohner nicht gewährleistet }\end{array}$ \\
\hline Dynamik & $\begin{array}{l}\text { Meist singuläres Schadensereignis ohne wesentliche Dynamik, } \\
\text { jedoch rasche Änderungen des Patientenzustands möglich. } \\
\Rightarrow \text { Wiederholte Nachsichtungen in kurzen Zeitabständen } \\
\Rightarrow \text { Ggf. Soforttransporte, sofern Patient nicht stabilisierbar }\end{array}$ & $\begin{array}{l}\text { Schadenslage dynamisch durch neue oder progrediente klinische } \\
\text { Symptomatik sowie Ausbreitung der Infektion. Patientenzustand } \\
\text { weniger dynamisch. } \\
\Rightarrow \text { Nachsichtungen in größeren Abständen über einen längeren } \\
\text { Zeitraum } \\
\Rightarrow \text { Soforttransporte seltener notwendig }\end{array}$ \\
\hline Aufgaben & $\begin{array}{l}\text { Notfallmedizinische Routineaufgaben, ggf. Massenanfallsversor- } \\
\text { gung mit kurzfristigem Abweichen von individualmedizinischen } \\
\text { Standards }\end{array}$ & Wie bei MANV. Zusätzlich ggf. Übernahme pflegerischer Tätigkeiten \\
\hline Sichtung & $\begin{array}{l}\text { Etablierte, evaluierte Vorsichtungsalgorithmen. Zeitbedarf unter } \\
\text { einer Minute pro Patient (von individueller SK abhängig; [16, } \\
\text { 28]). SK IV nur im absoluten Ausnahmefall }\end{array}$ & $\begin{array}{l}\text { Modifikation des Sichtungsvorgangs notwendig (Pulsoxymetrie und } \\
\text { Körpertemperatur; neurologische Komponenten ggf. nicht aussa- } \\
\text { gekräftig). Patientenwille kann zur SK IV führen. Höherer Zeitbedarf }\end{array}$ \\
\hline $\begin{array}{l}\text { Belastung } \\
\text { der Einsatz- } \\
\text { kräfte }\end{array}$ & Psychische Belastung dominiert & $\begin{array}{l}\text { Neben psychischem Stress (u.a. Angst vor eigener Ansteckung) zu- } \\
\text { sätzliche Belastung durch Tragen der Schutzausrüstung }\end{array}$ \\
\hline
\end{tabular}

Tab. 2 Phasenmodell des MANI-Managements in stationären Pflegeeinrichtungen im Kontext der COVID-19-Pandemie

\begin{tabular}{|c|c|c|c|}
\hline & Zeithorizont & Maßnahmen & Zuständigkeiten \\
\hline \multirow{4}{*}{$\begin{array}{l}\text { Phase 1: } \\
\text { akute Gefah- } \\
\text { renabwehr }\end{array}$} & \multirow[t]{4}{*}{ Stunden } & (Vor-)Sichtung & \multirow[t]{4}{*}{ San-EL/Rettungsdienst } \\
\hline & & Kritische Patienten stabilisieren & \\
\hline & & Akuten Hospitalisierungsbedarf feststellen & \\
\hline & & Kliniktransporte organisieren & \\
\hline \multirow{6}{*}{$\begin{array}{l}\text { Phase 2: } \\
\text { Übergangs- } \\
\text { betrieb si- } \\
\text { cherstellen }\end{array}$} & \multirow[t]{6}{*}{ Stunden - Tage } & Entscheidung über Evakuierung von Gesunden und/oder Infizierten & \multirow{6}{*}{$\begin{array}{l}\text { Heimleitung/Träger/San-EL/ } \\
\text { Kreisverwaltungsbehörde } \\
\text { (FüGK, ÖGD, Heimaufsicht)/ } \\
\text { Fahrdienste }\end{array}$} \\
\hline & & Absonderungsmaßnahmen sicherstellen & \\
\hline & & Infektionsüberwachung gewährleisten & \\
\hline & & Pflegerische Übergangsbetreuung sicherstellen & \\
\hline & & $\begin{array}{l}\text { Ambulante medizinische Versorgung sicherstellen (ärztliche Versorgung, Medika- } \\
\text { mente, Heilmittel) }\end{array}$ & \\
\hline & & Ggf. Transporte i. R.d. Evakuierung organisieren & \\
\hline \multirow{6}{*}{$\begin{array}{l}\text { Phase 3: } \\
\text { Regelbetrieb } \\
\text { wiederher- } \\
\text { stellen }\end{array}$} & \multirow[t]{6}{*}{ Tage - 1 Woche } & Pflegerische Regelbetreuung wiederherstellen & \multirow{6}{*}{$\begin{array}{l}\text { Heimleitung/Träger/ } \\
\text { Kreisverwaltungsbehörde } \\
\text { (FüGK, ÖGD, Heimaufsicht)/ } \\
\text { Fahrdienste }\end{array}$} \\
\hline & & Ambulante medizinische Versorgung sicherstellen & \\
\hline & & Absonderungsmaßnahmen konsequent fortführen & \\
\hline & & Infektionsüberwachung gewährleisten & \\
\hline & & Rücktransporte von Evakuierten organisieren & \\
\hline & & Reserven schaffen (Personal, Material) & \\
\hline
\end{tabular}

SK II). Bei reduzierten Aufnahmekapazitäten müssen diese auf 3 Zielkliniken verteilt werden, was bis $17.30 \mathrm{Uhr}$ abgeschlossen ist.

Parallel wird durch die FüGK festgestellt, dass der Träger für die nächsten $24 \mathrm{~h}$ nicht in der Lage ist, die Einrichtung personell zu besetzen. Aufgrund der großen Anzahl erkrankter Bewohner besteht zudem ein deutlich erhöhter Betreuungsbedarf. Mithilfe eines privaten ambulanten
Pflegediensts kann schließlich der Personalbedarf bis zum nächsten Nachmittag gedeckt und eine Evakuierung des Heims abgewendet werden.

\section{Tag 2 (Freitag)}

Eine halbe Stunde vor der geplanten Übergabe teilt der Einrichtungsträger mit, dass die Übernahme des Betriebs nicht wie vereinbart erfolgen kann. Die FüGK entscheidet sich daraufhin er- neut zur Alarmierung der San-EL. Vor Ort werden eine neuerliche Sichtung durchgeführt und wiederum 2 akute Kliniktransporte mittels RTW organisiert. Notdürftig kann, u.a. durch Unterstützung einer Hilfsorganisation und weiterer Freiwilliger, eine pflegerische Besetzung für die Nacht auf die Beine gestellt werden. 


\section{Tag 3 (Samstag)}

Die FüGK entscheidet, den Betrieb im Rahmen einer Ersatzvornahme dem privaten Pflegedienst interimsweise zu übertragen. Vor der Übergabe an diesen am Abend erfolgt eine dritte Sichtungsrunde durch den Leitenden Notarzt (LNA) mit 2 weiteren resultierenden Klinikeinweisungen.

\section{Tag 4 (Sonntag)}

Der LNA führt über seine vor Ort befindliche Allgemeinarztpraxis die ärztliche Betreuung der Bewohner fort und verordnet insbesondere erforderliche Dauer- und Bedarfsmedikation, die durch eine lokale Apotheke ausgegeben wird. Abends kann die Einrichtung wieder in die reguläre ambulante Versorgung übergeben und der Einsatz endgültig abgeschlossen werden.

\section{Abgrenzung MANV vs. MANI}

In der Literatur wird das Management von traumatisch bedingten Großschadenslagen umfassend behandelt [6, 18, 19], während sich zum Thema notfallmedizinische Einsatztaktik bei MANE/MANI keine spezifischen Einsatzalgorithmen finden. Gängige Sichtungskonzepte sind überwiegend für MANV-Lagen erstellt und evaluiert und können nicht ohne weiteres auf SARS-CoV-2-Infektionen übertragen werden. Die aktuelle Pandemiesituation stellt zudem erweiterte Anforderungen an die Einsatztaktik, die bei bisherigen Planungen nicht berücksichtigt wurden. Wesentliche Unterschiede zwischen MANV- und MANI-Lagen sind in - Tab. 1 zusammengefasst.

\section{Zielsetzung MANI-Management}

Das MANI-Management in Rahmen der COVID-19-Pandemie geht über die akute Gefahrenabwehr hinaus und dient unter anderem dazu, ohnehin schon an der Grenze der Belastbarkeit agierende Krankenhäuser von vermeidbaren Patientenaufnahmen $\mathrm{zu}$ entlasten und eine Infektionsverschleppung in andere Einrichtungen zu verhindern. Wann immer möglich sollte die Evakuierung betroffener Einrichtungen trotz der Schwächung

Med Klin Intensivmed Notfmed 2022 · 117:289-296 https://doi.org/10.1007/s00063-021-00816-1 () Der/die Autor(en) 2021

W. Schreiber · P. Wolf $\cdot$ N. Bigalke · M. U. Bigalke · B. M. Graf · M. S. Dittmar

\section{Managementstrategie für den Massenanfall von Erkrankten/Infizierten in Alten- und Pflegeheimen im Kontext der COVID-19-Pandemie}

\section{Zusammenfassung}

Hintergrund. Während der Corona Virus Disease-2019(COVID-19)-Pandemie sind gehäuft Ausbrüche in stationären Pflege- und Betreuungseinrichtungen zu beobachten, die die ambulanten Strukturen überfordern und zu einer rettungsdienstlichen Großschadenslage aufwachsen. Standardisierte einsatztaktische Vorgaben wie für den Massenanfall von Verletzten (MANV) fehlen. Methoden. Anhand eines konkreten Fallberichts und der Literatur stellen die Autoren eine Managementstrategie für den Massenanfall von Erkrankten bzw. Infizierten (MANE/MANI) während der Severe Acute Respiratory Syndrome Corona Virus-2(SARSCoV-2)-Pandemie vor und grenzen diese zur MANV-Einsatztaktikab.

Ergebnisse. Das Vorgehen bei MANI lässt sich in 3 Phasen einteilen und beginnt mit der akuten Gefahrenabwehr mit Sichtung, Stabilisierung kritischer Patienten und Transport der hospitalisierungspflichtigen Patienten. In Phase 2 werden die Betriebsbereitschaft der Einrichtung gesichert oder die Bewohner anderweitig untergebracht, falls das Personal in relevantem Ausmaß infiziert oder in Quarantäne ist. Die 3. Phase markiert die Rückkehr zum Regelbetrieb.

Diskussion. Phase 1 orientiert sich an MANV-Grundsätzen, Phase 2 am Krankenhauskrisenmanagement. Die Vermeidung einer Evakuierung der Bewohner zur Entlastung der Krankenhäuser stellt dabei ein wichtiges Einsatzziel dar. Mangelnde Einsatz- und Übungspraxis mit derartigen Lagen, die begrenzte Anwendbarkeit von etablierten Vorsichtungsalgorithmen sowie der hohe Koordinationsbedarf stellen die Führungskräfte vor Herausforderungen. Schlussfolgerung. Das vorgestellte Phasenmodell stellt einen praktikablen, ganzheitlichen Ansatz zum erweiterten notfallmedizinischen Management von MANI-Einsatzlagen dar.

Schlüsselwörter

SARS-CoV-2 - Massenanfall von Infizierten - Alten- und Pflegeeinrichtungen . Rettungsdienst $\cdot$ Krisenmanagement

\section{Management of COVID-19 mass casualty incidents in nursing and retirement homes}

\section{Abstract}

Background. During the coronavirus disease 2019 (COVID-19) pandemic, outbreaks in inpatient care facilities, which grow into a large-scale emergency scenario, are frequently observed. A standardized procedure analogous to algorithms for mass casualty incidents $(\mathrm{MCl})$ is lacking.

Methods. Based on a case report and the literature, the authors present a management strategy for infectious $\mathrm{MCl}$ during the severe acute respiratory syndrome coronavirus 2 (SARS-CoV-2) pandemic and distinguish it from traumatic $\mathrm{MCl}$ deployment tactics. Results. This management strategy can be divided into three phases, beginning with the acute emergency response including triage, stabilization of critical patients, and transport of patients requiring hospitalization. Phase 2 involves securing the facility's operational readiness, or housing residents elsewhere in case staff are infected or quarantined to a relevant degree. Phase 3 marks the return to regular operations.

Discussion. Phase 1 is based on usual $\mathrm{MCl}$ principles, phase 2 on hospital crisis management. Avoiding evacuation of residents to relieve hospitals is an important operational objective. The lack of mission and training experience with such situations, the limited applicability of established triage algorithms, and the need to coordinate a large number of participants pose challenges.

Conclusion. This strategic model offers a practical, holistic approach to the management of infectious mass casualty scenarios in nursing facilities.

\section{Keywords}

Mass casualty incidents · Emergency medical services - Residential facilities - Disease outbreaks · SARS-CoV-2 
der Personaldecke durch Erkrankung oder Quarantänepflicht vermieden werden. Dies findet beim nachfolgenden Phasenmodell (『Tab. 2) Berücksichtigung.

\section{Phase 1: akute Gefahrenabwehr}

Die Phase 1 orientiert sich weitgehend an den bei MANV-Lagen üblichen Einsatzgrundsätzen. Nach einem ersten Lageüberblick und Kräfteabgleich werden anhand einer (Vor-)Sichtung der sofortige Behandlungs- und Transportbedarf festgestellt und entsprechende rettungsdienstliche Ressourcen zugeteilt. Im Anschluss werden weniger kritische, aber dennoch hospitalisierungspflichtige Patienten identifiziert und deren Klinikaufnahme organisiert. Besonderes Augenmerk wird auf die Sicherheit der Einsatzkräfte gelegt, die insbesondere durch fachgerechte Durchführung von Barrieremaßnahmen (persönliche Schutzausrüstung, Desinfektion) gewährleistet wird. Bei längerdauernden Einsätzen muss die Phase 1 unter Umständen mehrfach durchlaufen werden (s. Fallbeispiel).

\section{(Vor-)Sichtung und Versorgung kritischer Patienten}

Im Rahmen einer nichtärztlichen Vorsichtung mittels starrer Algorithmen oder einer ersten ärztlichen Sichtung werden Patienten mit akuter Störung der Vitalfunktionen und sofortigem Behandlungsbedarf (SK I, „rot", Sofortbehandlung) identifiziert [9]. Bei der Vorsichtung wird man aus pragmatischen Erwägungen häufig nicht umhinkommen, den jeweiligen für den MANV-Fall etablierten Vorsichtungsalgorithmus anzuwenden, auch wenn dieser regelhaft nur bedingt für internistische/infektiologische Krankheitsbilder geeignet ist. Umso wichtiger ist die nachfolgende ärztliche Sichtung, die spätestens im Rahmen der Entscheidungsfindung über den Hospitalisierungsbedarf (s. unten) erfolgt. Neben den rein medizinischen Kriterien sollte auch das Vorhandensein eventueller Patientenverfügungen berücksichtigt werden, sodass bei schwerstem Verlauf und gleichzeitiger Ablehnung einer intensivmedizinischen Therapie auch die Einordnung in SK IV (palliative Behandlung) zu erwägen ist.

Im obigen Fallbeispiel wurde die erste Sichtung nicht nach dem Vorsichtungsalgorithmus, sondern nach der Entscheidungshilfe Hospitalisierung der Ärztlichen Leiter Rettungsdienst (ÄLRD) Bayern $[23,27]$ vorgenommen und um die Messung von Vitalparametern und Temperatur ergänzt. Dies führte, zusammen mit den notwendigen Hygienemaßnahmen, zu einem unerwartet hohen Zeitbedarf (über 6 min pro Patient).

In SK I gesichtete Patienten werden schnellstmöglich notfallmedizinisch stabilisiert und, sobald entsprechende Transport- und Aufnahmekapazitäten bereitgestellt wurden, in geeignete Krankenhäuser verbracht. Die Klinikzuweisung erfolgt analog zu den üblichen rettungsdienstlichen Vorgehensweisen mit entsprechender Voranmeldung (z. B. über die Leitstelle oder einen elektronischen Betten- und Behandlungskapazitätennachweis [14]). Soforttransporte, wie sie nach schweren Traumata mitunter notwendig sind, dürften die Ausnahme darstellen.

\section{Hospitalisierungsbedarf feststellen und realisieren}

Der Hospitalisierungsbedarf wird nach medizinischen Kriterien festgestellt und ist eine ärztliche Entscheidung, die auf Grundlage von entsprechenden Entscheidungshilfen getroffen werden kann $[13,23,27]$. Bei der Beurteilung kann die Einbeziehung von allgemeinmedizinischem Sachverstand bzw. die Abstimmung mit den weiterbetreuenden ambulanten ärztlichen und pflegerischen Versorgungsstrukturen von Vorteil sein.

$\mathrm{Da}$ es sich bei diesem Patientenkollektiv nicht mehr um kritische Patienten handelt, kann die Bettenzuweisung unter Einbeziehung der zuständigen Führungsebenen für die übergeordnete Bettenkapazitätssteuerung erfolgen [11]. Die Durchführung der Transporte erfolgt durch den Rettungsdienst, ggf. unter Nutzung von Sonderfahrzeugen und Kohortentransporten, um die Einsatzmittel der Regelvorhaltung zu entlasten.
Phase 2: Übergangsbetrieb sicherstellen

Während bei präklinischen MANV-Lagen der Einsatz mit Abschluss der ersten Phase und Räumung der Einsatzstelle in der Regel abgeschlossen ist, stellt sich bei Ausbruchsgeschehen, wie im obigen Fallbeispiel dargestellt, ein nachfolgendes Problem: Wenn das Pflegepersonal der Einrichtung zu einem erheblichen Anteil selbst ausfällt, kann unter Umständen die Versorgung der verbliebenen Bewohner der Einrichtung nicht mehr gewährleistet werden. Erschwerend kommt hinzu, dass auch nichthospitalisierte COVID19-Erkrankte einen deutlich höheren Pflegeaufwand benötigen, sodass die reguläre Personalbesetzung gegebenenfalls nicht ausreicht. Dies führt im ungünstigsten Fall zu einer Evakuierung der gesamten Einrichtung verbunden mit der Verlegung einer größeren Anzahl COVID-19-(Verdachts-)Fällen in andere Einrichtungen (Pflegeeinrichtungen oder Krankenhäuser). In Zeiten ohnehin angespannter Belegungssituationen sollte dies tunlichst vermieden werden, was nur im Zusammenspiel von Träger bzw. Heimleitung, Kreisverwaltungsbehörden (Katastrophenstab, öffentlicher Gesundheitsdienst [ÖGD], Heimaufsicht) und im Ausnahmefall der SanEL und des Katastrophenschutzes bewerkstelligt wird. Insbesondere in Bezug auf administrativ-organisatorische Weichenstellungen (Personaluntergrenzen etc.) ist eine enge Abstimmung mit der Aufsichtsbehörde, in diesem Fall der Heimaufsicht, anzustreben.

\section{Entscheidung über Evakuierung}

Für die Entscheidung, in welchem Umfang ein Weiterbetrieb der Einrichtung möglich gemacht werden kann bzw. ob die (Teil-)Evakuierung von Gesunden und/oder Erkrankten bzw. Infizierten erfolgen muss, können die Kriterien in - Tab. 3 sowie DAbb. 1 herangezogen werden.

\section{Pflegerische Übergangsbetreuung sicherstellen}

Sofern die pflegerische Betreuung nicht durch das verbliebene heimeigene Personal geleistet werden kann, muss dies 
Tab. 3 Entscheidungskriterien bezüglich der Evakuierung von Gesunden und/oder Erkrankten/Infizierten (in absteigender Wichtigkeit)

Pflegerische Betreuung sichergestellt? Krankenhauskapazität vorhanden? Sonstige Übernahmemöglichkeiten vorhanden? Absonderungsmöglichkeiten vorhanden oder zeitgerecht organisierbar (Räumlichkeiten, PSA, Hygieneartikel)?

Ambulante medizinische Betreuung gewährleistet?

Transportkapazität vorhanden?

anderweitig organisiert werden. Größere Träger können ggf. Pflegende aus anderen eigenen Einrichtungen zusammenziehen. Ansonsten ist zu prüfen, ob zur Unterstützung Freiwillige (z.B. aus dem Pflegepool der Vereinigung der Pflegenden in Bayern), Personal anderer Träger, Ehrenamtliche der Hilfsorganisationen oder Bundeswehrangehörige (nur im Katastrophenfall) rekrutiert werden können. Auch ist der Einsatz von asymptomatischem infiziertem Pflegepersonal nach den Maßgaben des Robert Koch-Instituts zu erwägen. Im Extremfall kann der Betrieb übergangsweise an einen anderen, leistungsfähigen $\mathrm{Be}$ treiber übergeben werden. Ein ausreichender Fachkräfteanteil muss jeweils sicherstellt sein, der jedoch kurzfristig die vorgeschriebene Fachkraftquote unterschreiten kann.

\section{Absonderungsmaßnahmen und Infektionsüberwachung sicherstellen}

Für die verbleibenden Bewohner sind in Absprache mit dem ÖGD die notwendigen Absonderungsmaßnahmen (Isolation, Quarantäne) sicherzustellen. Hierfür müssen gewisse personelle und bauliche Anforderungen erfüllt sowie ausreichend Hygienematerial (persönlicheSchutzausrüstung wie Schutzmasken, -brillen und -anzüge, Desinfektionsmittel) verfügbar sein. Es ist häufig sinnvoll, positiv getestete Bewohner in einen abgetrennten Bereich zusammenzulegen, um diese räumlich von den Gesunden $\mathrm{zu}$ isolieren. Durch eine engmaschige strukturierte klinische Überwachung der Bewohner kann auf neu symptomati- sche oder sich klinisch verschlechternde Patienten rechtzeitig reagiert werden.

\section{Ambulante medizinische Versorgung sicherstellen}

Neben der pflegerischen ist auch die ambulante hausärztliche Betreuung von großer Bedeutung für die Betriebsbereitschaft der Einrichtung. Diese kann u. a. durch Arbeitsüberlastung oder eigene Krankheits- bzw. Quarantänefälle der ambulanten Arztpraxen gefährdet sein. Ebenso ist auch die Versorgung mit Arznei- und Heilmitteln (insbesondere Sauerstoff) zu gewährleisten.

In Bayern wurde die Sicherstellung der ambulanten ärztlichen Versorgung im Rahmen des Krisenfalls auf der Ebene der Kreisverwaltungsbehörden in die Hände von eigens bestellten Versorgungsärzten [3] bzw. koordinierenden Ärzten [5] gelegt. Bewährt hat sich die interimsweise Beauftragung von Heimärzten, die sich um die Betreuung sämtlicher Bewohner kümmern. Die freie Arztwahl ist jedoch zu gewährleisten; insbesondere können keine Besuche anderer Ärzte ausgeschlossen werden.

\section{Transporte im Rahmen der Evakuierung organisieren}

Sofern die Entscheidung zugunsten einer (Teil-)Evakuierung gefallen ist, müssen die entsprechenden Transporte organisiert werden. Bewohner, die aufgrund ihres klinischen Zustands nicht auf einen qualifizierten Krankentransport angewiesen sind, sollten möglichst mit anderweitigen Fahrdiensten befördert werden, um den Rettungsdienst zu entlasten. Ist dies nicht möglich, sind Kohortentransporte zu erwägen.

\section{Phase 3: Regelbetrieb wiederherstellen}

Träger und Leitung der Einrichtung sind gefordert, in Abstimmung mit dem ÖGD und der Heimaufsicht so rasch wie möglich die Voraussetzungen für die Wiederaufnahme des Regelbetriebs zu schaffen. Neben einem funktionierenden Dienstplan mit eigenem Pflegepersonal sind die ambulante medizinische Versorgung sowie angeordnete Absonderungsund Infektionsüberwachungsmaßnah- men (Symptomverfolgung, Nachtestungen) sicherzustellen.

Im Anschluss kann sukzessive mit dem Rücktransport der ausgelagerten bzw. entlassfähigen Bewohner begonnen werden. Bezüglich der Transporte sollten o.g. Prinzipien beachtet werden.

Auch den eingesetzten Kräften des Rettungsdiensts sollte trotz stringenter Barrieremaßnahmen eine Selbstüberwachung auf Symptome und eine Virustestung angeraten und ermöglicht werden.

\section{Diskussion}

Die SARS-CoV-2-Pandemie stellt einen globalen Gesundheitsnotstand dar und sprengt gängige Schweregradeinteilungen von Großschadenslagen und Katastrophen [10]. COVID-19-Ausbrüche in stationären Pflegeeinrichtungen sind ein häufiges Phänomen während der Pandemie. Die hier dargestellte Einsatzstrategie stellt einen praktikablen, ganzheitlichen Ansatz zum erweiterten notfallmedizinischen Management solcher Lagen dar.

Die erste Phase lehnt sich dabei an die bekannten Einsatzgrundsätze der MANV-Versorgung an, sodass die Anwendung für die rettungsdienstlichen Einsatz- und Führungskräfte kein wesentliches Abweichen von etablierten Standards bedeutet. Dagegen orientiert sich Phase 2 mehr an Strategien des pandemiebezogenen Krankenhauskrisenmanagements mit den Stellgrößen Personal, Ressourcen und Raum [29-31]. Hier steht das Ziel im Vordergrund, in der betroffenen Pflegeeinrichtung mindestens das Betreuungsniveau der kompensierten Krisenversorgung zu erreichen, um den Betrieb aufrechterhalten zu können.

Ein vergleichbares Phasenmodell wurde bei einem Ausbruch in einem kanadischen Pflegeheim im Rahmen eines COVID-19-Ausbruchs angewendet. Hier erfolgte die organisatorische Unterstützung jedoch nicht durch den Rettungsdienst, sondern durch ein Krankenhaus [26].

Anders als bei den häufigeren MANVLagen kann der Rettungsdienst zu diesen speziellen MANI-Situationen wenig Übungs- und Praxiserfahrung vorweisen 


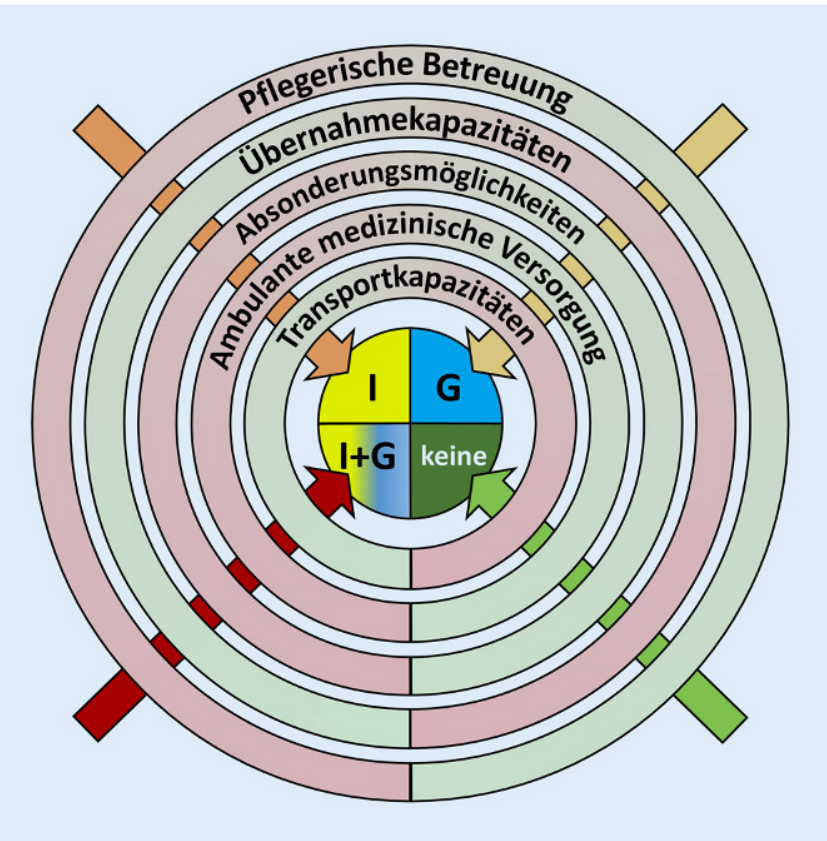

Abb. 1 \ Entscheidungshilfe über die Evakuierung gemäß der Kriterien aus • Tab. 3 (Kreissegmente) mit von außen nach innen abnehmender Relevanz. Die in den Kreissegmenten dargestellten Aspekte gehen fließend von der Regelsituation in eine zunehmende Mangelsituation über. Im Zentrum steht die Evakuierungsentscheidung (keine Evakuierung, Evakuierung der Gesunden/Nichtinfizierten $(G)$, der Infizierten/Erkrankten $(I)$ oder von Gesunden und Infizierten $(I+G)$ ). Günstig aus Sicht des Krankenhauskapazitätenmanagements wäre der Verzicht auf eine Evakuierung (rechter unterer Quadrant), die ungünstigste Variante die Evakuierung von allen Bewohnern (linker unterer Quadrant), die oberen beiden Quadranten stellen Zwischenstufen dar. Es sind auch Teilevakuierungen der jeweiligen Bewohnergruppen denkbar

und es sind keine spezifischen einsatztaktischen Handlungsempfehlungen verfügbar. Ferner kann der Einsatz nicht in jedem Fall nach der ersten Einsatzphase als abgeschlossen betrachtet werden. Im Gegensatz zu MANI-Lagen außerhalb der Pandemie stellt das Erkennen der infektiösen Genese hingegen weniger Schwierigkeiten dar und die Einsatzkräfte sind in Bezug auf die Vorhaltung der erforderlichen Schutzausrüstung und den richtigen Umgang damit gerüstet. Anders als bei auf unklare biologische oder auf sonstige gefährliche Agenzien zurückzuführenden Gefahrenlagen sind zudem keine über die üblichen Hygienevorkehrungen hinausgehenden Dekontaminationsmaßnahmen erforderlich.

Während für traumatologische Großschadenslagen eine Reihe von konkreten Handlungsempfehlungen vorliegt $[6,18,19]$, sucht man diese für den MANE-/MANI-Fall vergeblich.

Die in der Bundesrepublik gängigen Vorsichtungsalgorithmen wurden überwiegend für chirurgische Patienten ent- wickelt und evaluiert $[2,7,12,16-18$, 22, 28] und zeigen bei internistischen Patienten eine geringere Treffsicherheit [1]. Lediglich das Primäre Ranking zur initialen Orientierung im Rettungsdienst (PRIOR; [8]) ist explizit auch für internistische Patienten vorgesehen und kann bei diesem Kollektiv rote Patienten sensitiver identifizieren, neigt jedoch zu relevanter Übertriage [1]. Während in der Traumasichtung die motorische Komponente des GCS die größte Vorhersagekraft für schwerverletzte Patienten aufweist [15], ist dieser Parameter bei vorbestehend kognitiv oder motorisch beeinträchtigen Patienten wenig aussagekräftig, was die Anwendbarkeit von üblichen Vorsichtungsalgorithmen einschränkt. Die Bedeutung der ärztlichen Sichtung sei nochmals hervorgehoben.

Bei fehlendem Behandlungswillen kann die Eingruppierung in die Sichtungskategorie IV mit nachfolgender palliativer Behandlung gerechtfertigt sein. Sofern eine entsprechend dokumentierte und kommunizierte Festlegung des
Patientenwillens im Sinne eines Advance Care Planing besteht, ist dies für den Akutfall von Vorteil [21].

Das vorgestellte Fallbeispiel stellt sicherlich einen Extremfall mit komplettem Zusammenbruch der Betriebsbereitschaft der Pflegeeinrichtung dar. Eine Evakuierung konnte nur durch einen langdauernden Einsatz der SanEL und ein intensives Zusammenwirken aller Beteiligten letztlich mithilfe eines externen Pflegeunternehmens verhindert werden. In den meisten Fällen wird sich die betroffene Institution jedoch unmittelbar von der ersten Akutphase wieder in den Regelbetrieb überführen lassen, ohne dass es einer Übergangsphase bedarf. Umgekehrt ist ebenso denkbar, dass es keines Einsatzes der San-EL bedarf, sondern die Phasen 2 und 3 durch Heimleitung, niedergelassene Ärzteschaft und ggf. ÖGD bewältigt werden. Die beschriebene Einsatztaktik könnte auch auf Ausbrüche in anderen Einrichtungen, wie in Krankenhäusern, Reha-Kliniken oder Justizvollzugsanstalten, angewendet werden.

In komplexen Lagen wie dieser sind eine standardisierte Führungsstruktur und ein eingespielter Führungsablauf unverzichtbare Voraussetzungen für das Gelingen des Einsatzes [10]. Einen erheblichen Vorteil stellte im Einsatzbeispiel der zum Einsatzzeitpunkt bereits ausgerufene Katastrophenfall dar, da die behördlichen Führungsstrukturen (FüGK) schon etabliert waren und steuernd eingreifen konnten. In einer Ad-hoc-Lage ohne Katastrophenfall ist die Einbeziehung einer örtlichen Einsatzleitung (ÖEL) nach Art. 15 Bayerisches Katastrophenschutzgesetz erwägenswert, die durch ihre weitreichenden Befugnisse eine Gesamtkoordination der einzelnen Akteure leisten kann.

\section{Schlussfolgerung}

Das vorgestellte Phasenmodell stellt das akute Management von COVID-19-Ausbrüchen in Alten- und Pflegeheimen dar und zeigt die Unterschiede zu MANVLagen auf. Insbesondere im Bereich der (Vor-)Sichtung muss ggf. von etablierten Konzepten abgewichen werden. Nach der Akutphase steht bei kritischer Ein- 
schränkung der Personaldecke der Weiterbetrieb der Einrichtung im Fokus der Bemühungen. In Bayern werden die Katastrophenstäbe in Zukunft durch einen Pflegeleiter FüGK verstärkt, der übergeordnete Koordinationsaufgaben im Krisenmanagement der Pflegeeinrichtungen übernehmen soll [4]. Außerhalb des Katastrophenfalls sollte eine ÖEL einbezogen werden, die im Idealfall seitens des örtlichen Einsatzleiters mit einer medizinisch versierten Person (LNA, Organisatorischer Leiter Rettungsdienst) besetzt wird. Die aus der weltumspannenden Infektionslage gewonnen Erkenntnisse stellen eine wichtige Basis für die zukünftige Ausrichtung von sowohl Katastrophenverhütung („mitigation“) als auch Vorbereitung („preparedness“) dar, um für die nächste globale Pandemie besser gerüstet zu sein [10].

\section{Fazit für die Praxis}

\section{- Rettungsdienst und Katastrophen- schutz sehen sich während der Corona Virus Disease-2019(COVID- 19)-Pandemie mit Massenanfallsla- gen von „Severe Acute Respiratory Syndrome Corona Virus-2"(SARS- CoV-2)-Infizierten in Alten- und Pflegeheimen konfrontiert. \\ - Hierbei muss von der üblichen Ein- satztaktik für Massenanfallslagen abgewichen werden. \\ - Die Vermeidung einer Evakuierung zur Entlastung der Krankenhäuser stellt ein wichtiges Einsatzziel dar.}

\section{Korrespondenzadresse}

\section{PD Dr. Michael S. Dittmar}

Klinik für Anästhesiologie, Universitätsklinikum Regensburg

Franz-Josef-Strauss-Allee 11, 93053 Regensburg, Deutschland

michael.dittmar@ukr.de

Funding. Open Access funding enabled and organized by Projekt DEAL.

\section{Einhaltung ethischer Richtlinien}

Interessenkonflikt. W. Schreiber, P. Wolf, N. Bigalke, M.U. Bigalke, B.M.Graf und M.S. Dittmar geben an, dass kein Interessenkonflikt besteht.
Für diesen Beitrag wurden von den Autoren keine Studien an Menschen oder Tieren durchgeführt. Für die aufgeführten Studien gelten die jeweils dort angegebenen ethischen Richtlinien.

Open Access. Dieser Artikel wird unter der Creative Commons Namensnennung 4.0 International Lizenz veröffentlicht, welche die Nutzung, Vervielfältigung, Bearbeitung, Verbreitung und Wiedergabe in jeglichem Medium und Format erlaubt, sofern Sie den/die ursprünglichen Autor(en) und die Quelle ordnungsgemäß nennen, einen Link zur Creative Commons Lizenz beifügen und angeben, ob Änderungen vorgenommen wurden.

Die in diesem Artikel enthaltenen Bilder und sonstiges Drittmaterial unterliegen ebenfalls der genannten Creative Commons Lizenz, sofern sich aus der Abbildungslegende nichts anderes ergibt. Sofern das betreffende Material nicht unter der genannten Creative Commons Lizenz steht und die betreffende Handlung nicht nach gesetzlichen Vorschriften erlaubt ist, ist für die oben aufgeführten Weiterverwendungen des Materials die Einwilligung des jeweiligen Rechteinhabers einzuholen.

Weitere Details zur Lizenz entnehmen Sie bitte der Lizenzinformation auf http://creativecommons.org/ licenses/by/4.0/deed.de.

\section{Literatur}

1. Heller AR, Salvador N, Frank M et al (2017) Diagnostische Güte von Vorsichtungsalgorithmen für den Massenanfall von Verletzten und Erkrankten. Anaesthesist 66(10):762-772. https://doi.org/10. 1007/s00101-017-0336-y

2. Ausschuss Ärztlicher Leiter Rettungsdienst Bayern (2014) Nichtärztliche Vorsichtung: Algorithmus für den Massenanfall verletzter oder chemisch intoxikierter Patienten ab dem Schulalter. http://www.aelrd-bayern.de/images/stories/pdf/ Empfehlung_AG_Massenanfall_Vorsichtung_1.1. pdf. Zugegriffen:20. Dez. 2020

3. Bayerisches Staatsministerium des Innern, für Sport und Integration, Bayerisches Staatsministerium für Gesundheit und Pflege (2020) Notfallplan Corona-Pandemie: Aufrechterhaltung derArztversorgung während desfestgestellten Katastrophenfalls. Gemeinsame Bekanntmachung der Bayerischen Staatsministerien des Innern, für Sport und Integration und für Gesundheit und Pflege vom 26. März 2020, Az. D4-2484-2-7 und Az. G35-G8060-2020/26-16. BayMBI 2020(157)

4. Bayerisches Staatsministerium des Innern, für Sport und Integration, Bayerisches Staatsministerium für Gesundheit und Pflege (2020) Unterstützung der pflegerischen Versorgung während der Corona-Pandemie Bekanntmachung des Bayerischen Staatsministeriums des Innern, für Sport und Integration und des Bayerischen Staatsministeriums für Gesundheit und Pflege vom 21. Dezember 2020, Az. G43-G8300-2020/3446-1. BayMBI 2020(772)

5. Bayerisches Staatsministerium für Gesundheit und Pflege (2020) Bekanntmachung zum Vollzug des Infektionsschutzgesetzes (IfSG) Aufrechterhaltung der Arztversorgung während der CoronaPandemie Bekanntmachung des Bayerischen Staatsministeriums für Gesundheit und Pflege vom 2. Dezember 2020, Az. G35e-G8060-2020/4870. BayMBI 2020(707)
6. Beck A, Bayeff-Filloff M, Kanz K-G et al (2005) Algorithmus für den Massenanfall von Verletzten an der Unfallstelle. Notfall Rettungsmed 8(7):466-473. https://doi.org/10.1007/s10049-005-0769-8

7. Benson M, Koenig KL, Schultz CH (1996) Disaster triage: START, then SAVE-a new method of dynamic triage for victims of a catastrophic earthquake. Prehosp Disaster med 11(2):117-124

8. Bubser F, Callies A, Schreiber J et al (2014) PRIOR: Vorsichtungssystem für Rettungsassistenten und Notfallsanitäter. Rettungsdienst 37(8):730-734

9. Bundesamt für Bevölkerungsschutz und Katastrophenhilfe (2020) 8. Sichtungs-Konsensus-Konferenz 2019. Protokoll. https://www. bbk.bund.de/SharedDocs/Downloads/BBK/DE/ Downloads/GesBevS/8_Sichtungs-KonsensusKonferenz.pdf?_blob=publicationFile. Zugegriffen:22. Dez. 2020

10. Coccolini F, Sartelli M, Kluger Y et al (2020) COVID19 the showdown for mass casualty preparedness andmanagement:the Cassandra Syndrome. World J Emerg Surg 15(1):26. https://doi.org/10.1186/ s13017-020-00304-5

11. Dittmar MS, Altmeppen J, Bigalke MU et al (2021) Der Ärztliche Leiter Führungsgruppe Katastrophenschutz als zentrale Entscheidungsinstanz bei der Steuerung regionaler Krankenhauskapazitäten in der Pandemie. Ein Erfahrungsbericht zur ersten Welle der COVID-19-Pandemie in einem bayerischen Regierungsbezirk. Anaesthesist. https://doi.org/10.1007/s00101-020-00911-6

12. Dittmar MS, Bigalke M, Brunner A et al (2013) Ein regional angepasstes Vorgehen zur Vorsichtung und Sichtungskennzeichnung beim Massenanfall von Verletzten. Notarzt 29(06):253-259. https:// doi.org/10.1055/s-0033-1349617

13. Fistera D, Risse J, Manegold R et al (2020) COVID-19-Triage. Wer bleibt stationär? Das Modell Essen (COVID-19 Triage: Who is an inpatient? The Essen triage model). Dtsch Med Wochenschr 145(15):e87-e92. https://doi.org/10. 1055/a-1167-7596

14. Flemming A, Hoeper MM, Welte T et al (2020) Rettungsdienst. Schneller in die richtige Klinik. Dtsch Arztebl Int 117(24):A-1206

15. Garner A, Lee A, Harrison Ketal (2001) Comparative analysis of multiple-casualty incident triage algorithms. Ann Emerg Med 38(5):541-548. https://doi.org/10.1067/mem.2001.119053

16. Gutsch W, Huppertz T, Zollner C et al (2006) Initiale Sichtung durch Rettungsassistenten. Notfall Rettungsmed 9(4):384-388. https://doi.org/10. 1007/s10049-006-0827-x

17. Hiereth KI, Hornburger $\mathrm{P}$, Eyer $\mathrm{F}$ et al (2013) mSTaRT Trauma \& Intox. Notfall Rettungsmed 16(8):627-636. https://doi.org/10.1007/s10049013-1792-9

18. Kanz KG, Hornburger P, Kay MV et al (2006) mSTaRTAlgorithmus für Sichtung, Behandlung und Transport bei einem Massenanfall von Verletzten. Notfall Rettungsmed 9(3):264-270. https://doi. org/10.1007/s10049-006-0821-3

19. Kippnich M, Schmitz M, Schmidt J et al (2019) Massenanfall von Verbrennungspatienten. Notfall Rettungsmed 22(4):284-290. https://doi.org/10. 1007/s10049-018-0555-z

20. Mas Romero M, Avendaño Céspedes A, Tabernero Sahuquillo MT et al (2020) COVID-19 outbreak in long-term care facilities from Spain. Many lessons to learn. PLoS ONE 15(10):e241030. https://doi. org/10.1371/journal.pone.0241030

21. Michels G, Heppner H-J (2020) Priorisierung von Pflegeheimbewohnern aus notfallmedizinischer Sicht. Corona-Pandemie 2020 (Prioritization of 
geriatric patients in care homes and residential homes in the context of the COVID pandemic). MMW Fortschr Med 162(9):48-51. https://doi.org/ 10.1007/s15006-020-0477-9

22. Paul A, Kay M, Huppertz T et al (2009) Validierung der Vorsichtung nach dem mSTaRT-Algorithmus. Unfallchirurg 112(1):23-32. https://doi.org/10. 1007/s00113-008-1517-6

23. Rettungsdienstausschuss Bayern (2020) Entscheidungshilfe zur Patientenzuweisung bei Verdacht aufCOVID-19 für den bayerischen Rettungsdienst. http://www.aelrd-bayern.de/images/COVID19_ RD_202012.pdf.Zugegriffen: 15.Dez. 2020

24. Robert Koch Institut (2020) Täglicher Lagebericht des RKI zur Coronavirus-Krankheit-2019 (COVID-19). https://www.rki.de/ DE/Content/InfAZ/N/Neuartiges_Coronavirus/ Situationsberichte/Dez_2020/2020-12-21-de. pdf?_blob=publicationFile (Erstellt: 21. Dez. 2020). Zugegriffen:22. Dez. 2020

25. Sefrin P, Ging E (2006) Die Sanitätseinsatzleitung - ein praktikables Führungsinstrument? Notfall Rettungsmed 9(3):315-320. https://doi.org/10. 1007/s10049-006-0808-0

26. Stall NM, Farquharson C, Fan-Lun C et al (2020) A hospital partnership with a nursing home experiencing a COVID-19 outbreak. Description of a multiphase emergency response in Toronto, Canada.J Am Geriatr Soc 68(7):1376-1381. https:// doi.org/10.1111/jgs.16625

27. Thies N, Urban B, Kraus M et al (2021) Entscheidungshilfe zur Patientenzuweisung bei Verdacht auf COVID-19 für die Anwendung im bayerischen Rettungsdienst. Notfall Rettungsmed. https://doi. org/10.1007/s10049-021-00855-3

28. WolfP, Bigalke M, GrafBM et al (2014) Evaluation of a novel algorithm for primary mass casualty triage by paramedics in a physician manned EMS system: a dummy based trial. Scand JTrauma ResuscEmerg Med 22(1):50. https://doi.org/10.1186/s13049014-0050-6

29. Wurmb T, Ertl G, Ernestus R-I et al (2020) Command and control in hospitals during SARSCoV-2 pandemic: the windmill model of disaster response. J Emerg Manag 18(6):19-22. https://doi. org/10.5055/jem.2020.0520

30. Wurmb T, Scholtes K, Kolibay F et al (2020) Hospital preparedness for mass critical care during SARSCoV-2 pandemic. Crit Care 24(1):386. https://doi. org/10.1186/s13054-020-03104-0

31. Wurmb T, Scholtes K, Kolibay F et al (2020) Massenanfall kritisch kranker Patienten, „mass critical care" im Krankenhaus am Beispiel derSARSCoV-2 Pandemie. In: Bundesamt für Bevölkerungsschutz und Katastrophenhilfe (Hrsg) Handbuch Krankenhausalarm- und -einsatzplanung (KAEP). Vorab-Teilveröffentlichung anlässlich der COVID19-Pandemie. Bundesamt für Bevölkerungsschutz und Katastrophenhilfe, Bonn

\section{Aktuelle Buchempfehlungen aus dem Springer-Verlag}

\section{GOÄ 2022 Kommentar, IGeL-Abrechnung} Gebührenordnung für Ärzte

GOÄ 2022

Kommentar,

IGeL-Abrechnung

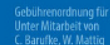

16. Alulage פ̂n Springer

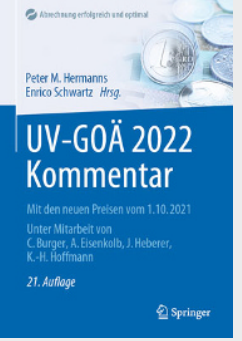

\section{UV-GOÄ 2022 Kommentar}

Mit den neuen Preisen vom 1.10.2021

Hermanns, Peter M., Schwartz, Enrico (Hrsg.)

XVII, 739 Seiten

2022, 21. Auflage

Springer-Verlag

ISBN 978-3-662-64487-4

$69,99 €$

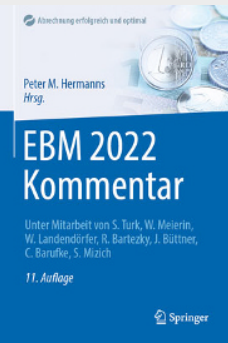

\section{EBM 2022 Kommentar}

Hermanns, Peter M. (Hrsg.)

XXVIII, 1004 Seiten

2022, 11. Auflage

Springer-Verlag

ISBN 978-3-662-64481-2

$79,99 €$

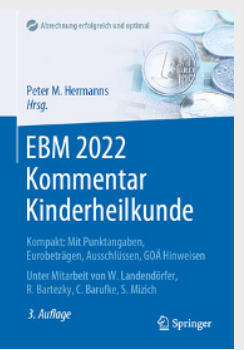

\section{EBM 2022 Kommentar Kinderheilkunde}

Kompakt: Mit Punktangaben, Eurobeträgen, Ausschlüssen, GOÄ Hinweisen

Hermanns, Peter M. (Hrsg.)

XVII, 375 Seiten

2022, 3. Auflage

Springer-Verlag

ISBN 978-3-662-64483-6

$44,99 €$ 